\title{
Automatic anatomical segmentation of the liver by separation planes
}

\author{
D. Boltcheva ${ }^{\mathrm{a}, \mathrm{b}, *}$, N. Passat ${ }^{\mathrm{b}, \mathrm{c}}$, \\ V. Agnus ${ }^{a}$, M.-A. Jacob-Da Col ${ }^{\text {b }}$, C. Ronse ${ }^{b}$, L. Soler ${ }^{a}$ \\ a IRCAD, Virtuals, 1 place de l'Hôpital, 67091 Strasbourg, France \\ ${ }^{\mathrm{b}}$ LSIIT, UMR 7005 CNRS-ULP, Strasbourg I University, France \\ ${ }^{\mathrm{c}} I P B$, UMR 7004 CNRS-ULP, Strasbourg I University, France
}

\begin{abstract}
Surgical planning in oncological liver surgery is based on the location of the liver segments and tumours inside these structures. The detection of the boundaries between the segments is then the first step of the preoperative planning. The proposed method, devoted to binary images of livers segmented from CT-scans, has been designed to delineate these segments. It automatically detects a set of landmarks using a priori anatomical knowledge and differential geometry criteria. These landmarks are then used to position planes delineating the 8 liver anatomical segments according to Couinaud's definition. The results of validations on 7 clinical cases tend to prove that the method is reliable for most of these separation planes.
\end{abstract}

Key words: computer assisted surgery, delineation planes, landmark detection, discrete differential geometry, operation planning.

\section{Introduction}

In the field of liver surgery, 3D visualization of the anatomical liver segments and their relationship to tumours are of major interest for the preoperative planning. For patients suffering from a malignant liver disease, tumour resection provides the best survival rate. To carry out this resection, surgeons use two operative methods: tumorectomy and segmentectomy. Tumorectomy is only used for so called peripheral tumours (located less than $5 \mathrm{~mm}$ away from

* Corresponding author.

Email address: dobrina.boltcheva@ircad.u-strasbg.fr (D. Boltcheva). 
the boundary of the liver and having a maximum diameter of $1 \mathrm{~cm}$ ). Segmentectomy addresses most lesions. It takes into account potential proliferation of tumours, which spread through the portal venous network. Therefore, the liver has been divided into several regions corresponding to vascular territories of the portal network. The segmentation proposed by Couinaud [1] is the current international reference. It divides the liver into 8 main regions, which can be recognized by using two types of landmarks: vascular landmarks and morphological landmarks that are visible on the liver surface. Anatomical segments are used as ablation units (see Fig. 1). However, preoperative localization of tumours inside these various segments from medical images is often a complex task.

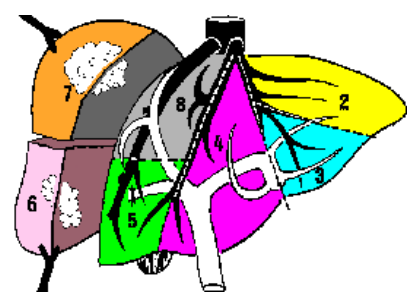

Fig. 1. Ablation of segments VI and VII of the liver.

We propose here an automatic method to delineate the liver classical segmental anatomy from 3D binary images. After a preliminary curvature estimation step, different pieces of medical knowledge are used to localize anatomical landmarks on the liver surface. The plane boundaries between the anatomical segments are then defined from these landmarks.

The paper is organised as follows. In Section 2, useful knowledge concerning liver anatomical structure and existing computer based methods for segment delineation are presented. In Section 3, background notions about anatomical landmarks and differential geometry concepts are introduced in order to justify the method detailed in Section 4. Experimental results are described and discussed in Section 5. Conclusion and perspectives are finally proposed in Section 6.

\section{State of the art}

\subsection{Liver segmental anatomy}

The liver is an active blood filter located on the trajectory of blood, between the intestine and the heart. It presents a complex internal anatomy, composed of hepatic cells surrounded by very dense vascular networks.

Morphologically, the liver lies in the abdominal cavity, and it is split into a 
large right and a small left lobe by the falciform (or suspensory) ligament extending from the anterior abdominal wall (see Fig. 2(a)).

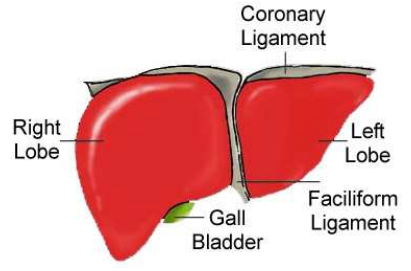

(a)

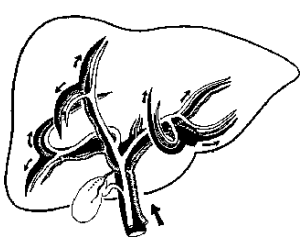

(b)

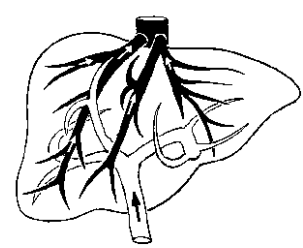

(c)

Fig. 2. (a) Left and right liver lobes. (b), (c): Vascular networks of the liver (arrows indicate the direction of blood flow): (b) portal vein (black), hepatic artery (grey) and biliary ducts (white); (c) portal vein (white) and hepatic veins (black).

Four different vessel systems supply and drain the liver: portal vein, hepatic vein, hepatic artery and biliary duct. Division of the portal inflow separates the liver in two parts, a right and left hemiliver, along the principal plane that passes through the gall bladder bed towards the vena cava (plane b on Fig. $3(\mathrm{a}))$.

The liver is supplied by the hepatic artery, which is close to the portal vein and the biliary network (see Fig. 2(b)). The hepatic venous network drains the blood coming from the portal vein after filtering. It is then located between the main branches of the portal network (see Fig. 2(c)). This position property has been used by Goldsmith and Woodburne [2] to divide the liver into 4 sectors corresponding to the 4 vascular territories of the portal vein, separated by the hepatic veins. The left hepatic vein separates the two sectors of the left hemiliver, while the right hepatic vein separates the two sectors of the right hemiliver. These sectors are: left lateral (LL), left paramedian (LP), right paramedian (RP) and right lateral (RL) (see Fig. 3(a)).

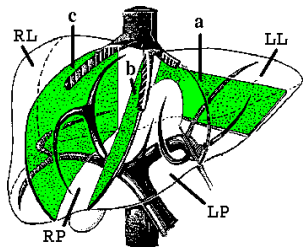

(a)

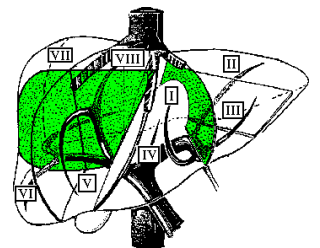

(b)

Fig. 3. (a) Goldsmith and Woodburne's definition of the 4 liver sectors from branches of the hepatic vein: left lateral (LL), left paramedian (LP), right lateral (RL) and right paramedian (RP). (b) Couinaud's definition of the 8 segments obtained by division of the 4 sectors.

According to Couinaud [1], the liver is composed of 8 independently supplied 
segments, representing the dispersion of the portal vein branches inside the 4 sectors (see Fig. 3(b)). Sectors of the right hemiliver are transversally split into segments by a plane passing through the axis of the portal vein right branch. The RP sector is subdivided into segment V (inferior) and segment VIII (superior) and the RL sector is subdivided into segment VI (inferior) and segment VII (superior). The LP sector is divided into two segments, III and IV, by a vertical plane that passes through the trace of the falciform ligament on the inferior border (or the umbilical fissure) towards the vena cava. The LL sector is the only one containing one segment, segment II. The spigelian lobe is a distinct anatomical segment and is labelled segment I. It receives branches of the portal network from the right and the left liver and drains independently into the vena cava.

It has to be noticed that the radiological delineation procedures are essentially based on the concept of three vertical planes that divide the liver into four main regions and of a transverse scissura that further subdivides the segments into two subsegments each (see Fig. 4). Moreover according to the previous definitions, most separation planes can be defined using external landmarks. These landmarks (vena cava, gall bladder bed, umbilical fissure or trace of the suspensory ligament on the inferior liver border) are generally used by surgeons to define segments before carrying out a surgical intervention.
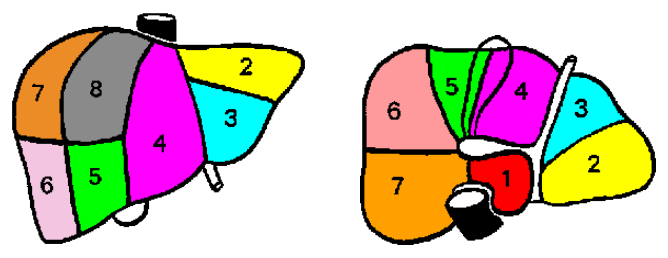

Fig. 4. Liver segments, according to Couinaud. Left: anterior view. Right: posterior view.

\subsection{Computer aided methods for segment delineation}

Several works have dealt with the development of software tools allowing to delineate the anatomical segments of the liver.

Two different approaches have been proposed. The first one consists in positioning planes that separate segments. To the best of our knowledge, there does not exist fully automated method enabling to place these planes since landmarks localization is a difficult task. Meinzer et al. [3] improve the computerbased preoperative planning system developed in Heidelberg [4] and propose a module for manual positioning of 10 landmarks on specific regions of the liver, such as the hepatic vein trunk (vena cava) and the main branches of the portal vein. These landmarks are then used to compute 3 vertical planes 
and 4 horizontal triangles. After positioning, the planes are manually adjusted in order to correctly fit the patient's 3D model. Osorio et al. [5] propose to manually place 8 landmarks on the scanner of the patient. These landmarks are localized on the left, right and median hepatic veins, on the inferior vena cava, on the bifurcation of the right portal vein, and at the boundary of the left liver. Boundaries between segments are then computed, and the various segments are identified and named.

The second approach proposes to compute vascular territories using the functional definition of the liver segments. It consists in labelling the portal vein branches and computing their influence regions. Meinzer et al. [3] also propose a module based on this strategy. However the labelling of branches remains interactive. Selle et al. [6,7] have developed a similar approach including automated labelling, taking bifurcation angles and vessel size into account. Nevertheless this labelling is not totally correlated with the conventional anatomical definition. Thus the resulting segments (and for instance the segment I) do not match the ones in anatomical atlases. Furthermore, the method only takes into account the bifurcations of the venous network, and does not use the global liver volumetry, which can induce errors that have to be manually corrected.

To meet both limitations, Soler et al. $[8,9]$ propose to take global volumetry into account for labelling, and to confront an anatomical atlas of liver segments with the segments that have been computed. During the segmentation and the labelling step the method generates more segments than the reference atlas. These segments are then merged during the confrontation step which allows an automatic correction. However, the confrontation can fail if the 3D model of the patient is too different from the atlas. Moreover the method does not use characteristic landmarks on the liver surface, which would enable to avoid the correlation errors.

We finally point out that all these methods use the liver venous network, and could thus fail if vein branches are not visible on the processed images. For instance if the images is acquired at the portal time, only the major branches of the portal vein can be extracted from the 3D enhanced helical CT-scans.

The method proposed in this paper consists in automating the plane placement method of Meinzer et al. and could also be used as a complement to the method of Soler et al. to take the patient's anatomy into account. Moreover, the automation is allowed by using surface mean curvature analysis for landmark localization. 


\section{Background}

This section presents the anatomical and theoretical background necessary to understand and justify the method described hereafter.

\subsection{Definition of anatomical landmarks}

The proposed method is based on the detection of six anatomical landmarks essentially located on the liver surface, and on the construction of five delineation planes between the liver segments. These landmarks, illustrated in Fig. 5 are defined as follows:

- A: superior part of the hepatic vena cava;

- B: inferior part of the hepatic vena cava;

- C: bed of the gall bladder;

- D: trace of the suspensory ligament;

- E: middle of the left lobe curve;

- F: centre of mass of the right liver.

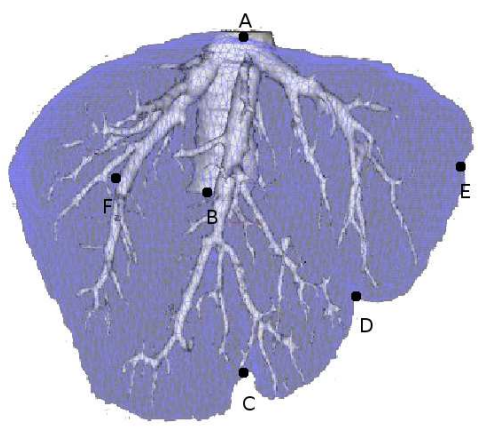

Fig. 5. Points used as landmarks (vena cava in white and liver in grey).

These points are chosen so that the anatomical definition of the following segments can be found.

- The $\mathrm{AB}$ axis represents the vena cava axis, while the point $\mathrm{C}$ is located on the gall bladder bed. Thus, the ABC plane splits the liver into left and right hemilivers.

- The point $\mathrm{D}$ is located on the inferior liver border and represents the passage of the suspensory ligament. Therefore, the ABD plane splits the liver into left and right liver lobes according to the morphological definition. Moreover, this plane delineates the segment IV inside the left hemiliver.

- The point $\mathrm{E}$ is located on the left liver lobe border and is used to divide the left hemiliver into sectors (LP and LL) according to the Goldsmith and 
Woodburne's definition. This enables the delineation of segment II from segment III inside the left hemiliver.

- The point $F$ represents the centre of mass of the right hemiliver. This point is used to define two delineating planes. The first one passes through this point towards the vena cava axis and corresponds to the separation between the RP and the RL sectors, while the second one is located at the level of the right branch of the portal vein and transversally splits the sectors into upper (VII and VIII) and lower (V and VI) segments.

In order to detect these landmarks, the proposed strategy is based on differential geometry criteria (mean surfacic curvature and $2 \mathrm{D}$ curvature of the liver).

\subsection{Curvature computation}

The mean surfacic curvature provides a characterization of the convexity or the concavity of a shape. In [10], Bullard et al. propose a method to assess, in each voxel of the surface of a discrete object, the value of its mean curvature. This method consists in computing the ratio of voxels belonging to the object within the neighbourhood of each voxel of its surface. The justification of the method lies in the fact that there exists an affine relation between the mean curvature of a point of the surface and the volume located inside the surface of a spherical sampling element centred in this point (see Fig. 6):

$$
H \approx \frac{4 V}{\pi b^{4}}-\frac{8}{3 b}
$$

where $H$ is the mean curvature, $b$ is the radius of the spherical neighbourhood of the point and $V$ the volume of the neighbourhood located inside the surface.

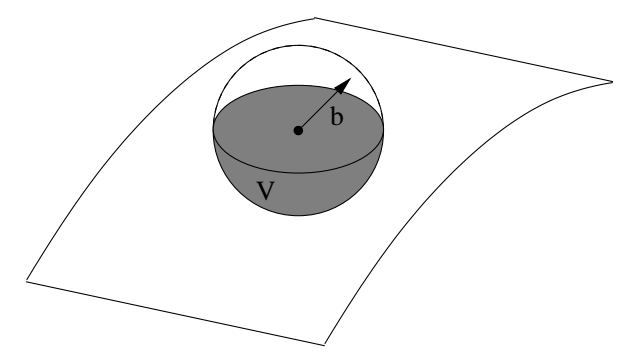

Fig. 6. Volume of the neighbourhood of a point of a surface located inside the latter. This volume $(V)$ is represented in grey. The radius of the spherical neighbourhood is denoted by $b$.

Fig. 7 illustrates the result of this approach applied on a binary liver image: dark areas are convex regions (i.e. negative mean curvature regions), while 


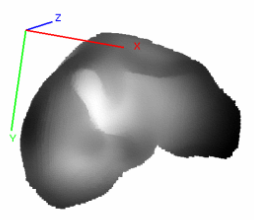

(a)

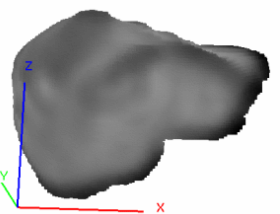

(b)

Fig. 7. Example of results obtained after applying the mean curvature estimation method on a discretized liver. (a) View of the inferior side. (b) View of the posterior side of the liver.

light areas correspond to concave regions (i.e. positive mean curvature regions). It has been experimentally observed that sampling elements with $3 \mathrm{~cm}$ radius provide satisfactory curvature estimations.

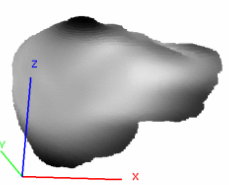

(a)

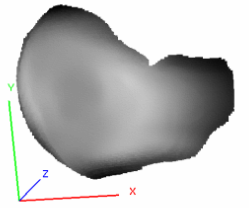

(b)

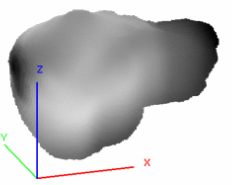

(c)

Fig. 8. Results of 2D curvature estimation. (a) Frontal view of the curvature obtained with the axial disk. The top and the bottom regions can be detected. (b) Top view of the curvature obtained with the coronal disk. The most convex region on the posterior liver border can be detected. (c) Frontal view of the curvature obtained with the sagittal disk. The left lobe and the right extremity can be localized.

However, the mean surfacic curvature information, computed with a spherical sampling element, can be insufficient to detect landmarks, since it only provides the overall shape of the liver surface. In order to obtain supplementary curvature information, a solution can consist in applying a similar method for $2 \mathrm{D}$ curvature estimation of curves obtained by intersecting the liver surface and each one of the three orthotropic planes (Oyz, Oxy and Oxz planes respectively represent the sagittal, axial and coronal planes). In this case, the considered sampling element is no longer a sphere but a disk. These new sampling elements allow a fast detection of some particular regions on the liver surface, such as the left lobe and the borders, thanks to their shape and specific localization (see Fig. 8).

The method used to successively position the landmarks and the separation planes is detailed in the following section. 


\section{Segment computation method}

\subsection{Input and output}

The method takes as input two isotropic binary images containing respectively the liver and the hepatic vein (which have to be correctly superimposed) and 2D and 3D liver curvature images computed with the previously described method. It provides as output a labelled image of the liver, each label corresponding to a segment according to the Couinaud's definition.

\subsection{ABC plane: separation of left and right hemiliver}

To divide the left hemiliver from the right hemiliver during a surgical intervention, surgeons use a vertical plane located in the axis of the trunk of the vena cava and passing through the bed of the gall bladder. The left hemiliver is composed of segments II, III and IV, whereas the right hemiliver contains segments V, VI, VII and VIII.

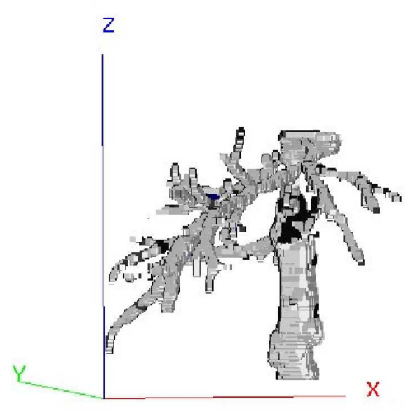

(a)

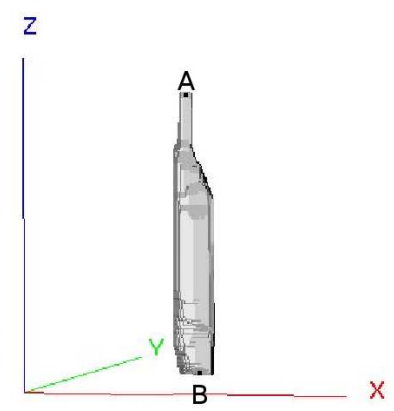

(b)

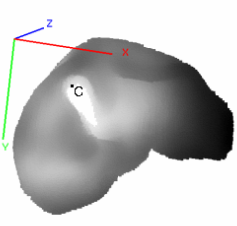

(c)

Fig. 9. (a) Hepatic vein. (b) Trunk of the vena cava. (c) Inferior view of the liver. In white, the trace of the gall bladder. Localization of the $\mathrm{C}$ point.

To compute this plane, called ABC, it is necessary to first localize the landmark points A, B and C (see Fig. 5).

In order to detect the trunk of the vena cava, a binary opening with a vertical and cylindrical structuring element is performed on the image of the hepatic vein. The radius of this structuring element has been fixed to 1 voxel whereas its height is adaptive to the shape size and varies from 50 to 10 voxels. This mathematical morphology operation enables to isolate the trunk of vena cava. Then, points A and B respectively correspond to the upper and lower part of 
this trunk.

The detection of the point $\mathrm{C}$ requires the localization of the gall bladder which presents a very high positive mean curvature. The curvature computed with the sagittal disc enables to detect it using a thresholding of the highest grey levels of the voxels on this face. The thresholding operation selects all points $q$ of the face such as Intensity $(q) \in\left[0.9 \times g_{\max }, g_{\max }\right]$, where $g_{\max }$ is the maximal curvature value of the face. The point $\mathrm{C}$ is then chosen as the closest point to the liver border, as illustrated in Fig. 9. The ABC plane finally built delineates both left and right hemilivers (see Fig. 10).

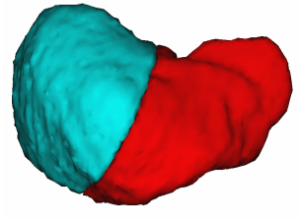

(a)

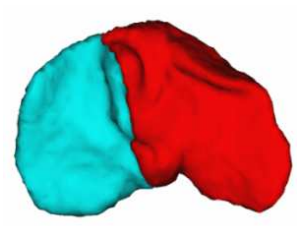

(b)

Fig. 10. Delineation of left and right liver. (a) Top view; (b) bottom view.

\subsection{ABD plane: delineation of segment IV}

From an anatomical point of view, segment IV is located in the left hemiliver and can be delineated by a vertical plane passing through the falciform ligament towards the vena cava (ABD plane). The point $\mathrm{D}$ corresponds to the trace of the suspensory ligament on the inferior liver border and has then to be detected (see Fig. 5).

The inferior border presents a very marked ridge interrupted in two points: on the left, by the suspensory ligament, and on the right, by the impression of the gall bladder. Therefore, it has a high negative mean curvature corresponding to a very convex shape, which is interrupted in two points where the mean curvature increases.

A preliminary step to the localization of the point D is the detection of the inferior border. This is done by scanning the mean curvature image coronal slices (in the $O x$ direction) to detect the point presenting the minimal curvature (see Fig.11(a)). In order to reduce the research area, the curvature is only computed in the region defined by:

$$
z \leq \operatorname{dim} Z-\frac{\operatorname{dim} Z}{\operatorname{dim} Y} \times y, \forall x
$$


where the inferior anterior border is necessarily located.

The selected points are then gathered by performing a binary dilation (see Fig.11(b)). Once the inferior border has been detected, it is used as a binary mask to only keep this border in the coronal curvature image. The point D is then chosen as being the leftmost voxel presenting the lowest curvature of the remaining area (see Fig.12(a)).

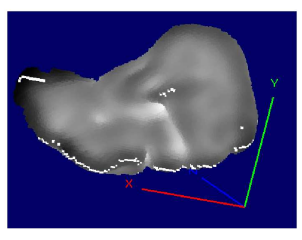

(a)

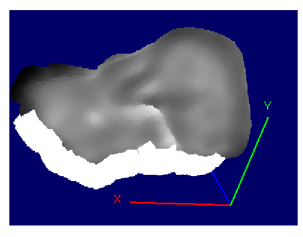

(b)

Fig. 11. Posterior view of the liver. (a) Curvature minima for each YZ half plane. (b) The largest connected component of the dilation corresponds to the inferior border.

The ABD plane finally obtained delineates segment IV inside the left hemiliver, as illustrated in Fig.12(b).

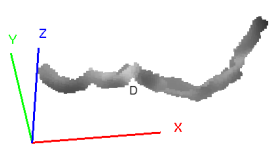

(a)

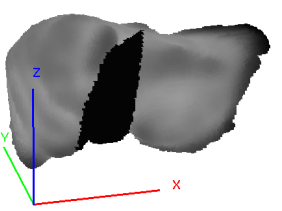

(b)

Fig. 12. (a) The inferior border on the coronal curvature image and the point $\mathrm{D}$ (corresponding to the trace of the suspensory ligament on this border). (b) Segment IV that has been automatically delineated by the method (black).

\subsection{ABE plane: delineation of segments II and III}

Segments II and III are the last two segments of the left hemiliver and represent the left liver lobe. Segment II corresponds of the LL sector and is located on the posterior border of the left liver lobe.

To delineate these segments, the ABE plane has to be computed by first detecting the point $\mathrm{E}$, located in the middle of the left lobe border.

The left lobe border is the most convex region of the liver, characterized by a very high negative mean curvature. The curvature image that has been 
computed with the sagittal disk enables to detect it by thresholding. This thresholding operation consists in selecting all voxels $q$, such as Intensity $(q) \in$ $\left[g_{\min }, g_{\min }+0.2 \times\left(g_{\max }-g_{\min }\right)\right]$ where $g_{\min }$ and $g_{\max }$ respectively correspond to the lowest and the highest curvature of the image. If several connected components are detected, the leftmost one is considered as being the searched component (see Fig. 13(a)).

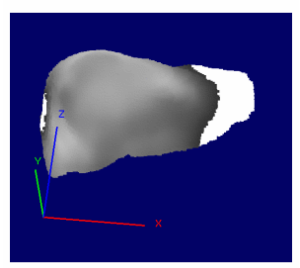

(a)

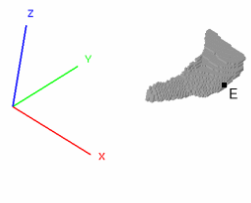

(b)

Fig. 13. (a) Anterior view of the thresholding result: the left liver lobe (white) is the leftmost connected component. (b) Location of the point $\mathrm{E}$ on the left liver lobe.

The centre of mass of the left lobe component finally provides the point E. Indeed, this point is located at the leftmost intersection of the parallel to the $O x$ axis starting from the centre of mass and the connected component of the left lobe (see Fig. 13(b)).

The resulting $\mathrm{ABE}$ plane which passes through the point $\mathrm{E}$ towards the vena cava separates segments II and III, as illustrated in Fig. 14(a). However, since the volume of the obtained segment II is generally smaller than the expected one, the separation is adjusted by moving the plane towards the liver center. This improvement consists in no longer considering points $\mathrm{A}$ and $\mathrm{B}$ on the vena cava, but the middle point of segments $\mathrm{AD}$ and $\mathrm{BD}$, respectively denoted by A' and B'. An example of such a delineation is illustrated in Fig. 14(b).

\subsection{ABF and HH'F planes: delineation of right hemiliver segments}

The right hemiliver is composed of segments V, VI, VII and VIII. From an anatomical point of view, there are no external landmarks enabling to delineate them. As a consequence, we propose to consider the centre of mass (point F), since its use ensures that the delineation will be performed through the middle of the right liver, where portal vein branches supplying each segment usually separate.

The method builds two planes from this point. The first one is the vertical plane, $\mathrm{ABF}$ generated by point $\mathrm{F}$ and $\mathrm{AB}$. The second one contains the point $\mathrm{F}$ and is normal to AB. This transversal plane is called HH'F. 


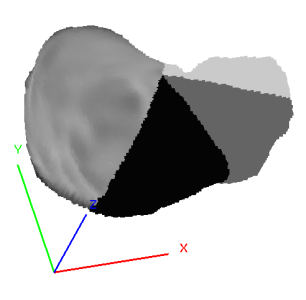

(a)

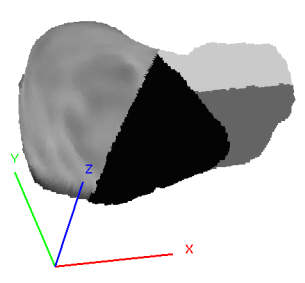

(b)

Fig. 14. Top view of left liver. (a) Before adjustment. (b) After adjustment of the ABE plane.

The vertical plane ABF separates both right hemiliver sectors according to the Goldsmith and Woodburne's definition, as illustrated in Fig. 15(a), while the transversal plane splits segments in each sector according to Couinaud's definition (see Fig. 15(b)).

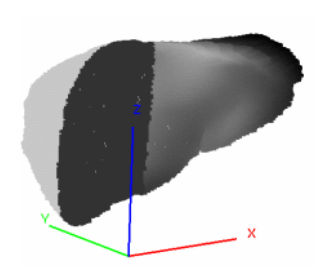

(a)

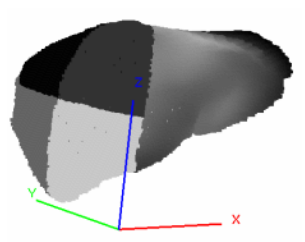

(b)

Fig. 15. (a) Vertical separation plane delimiting segments V and VIII from segments VI and VII. (b) Horizontal separation plane enabling to locate the 4 segments of the right liver.

\subsection{Delineation of segment I}

As segment I is part of both right and left hemilivers, it has to be treated separately from the other segments. It can be determined using two previously defined planes: $\mathrm{ABD}$ and $\mathrm{ABF}$. By respectively prolonging the $\mathrm{ABD}$ plane in the right hemiliver and the $\mathrm{ABF}$ plane in the left hemiliver, parts of segments previously assumed to belong to segments II, VI and VII are labelled as parts of segment I. An example of such a delineation is illustrated in Fig. 16. However, it has to be noticed that this approach can be inaccurate. Indeed, although the anatomical definition of this segment is only based on internal vascular landmarks, the proposed strategy only relies on knowledge concerning its position and adjacency according to its neighbours. 


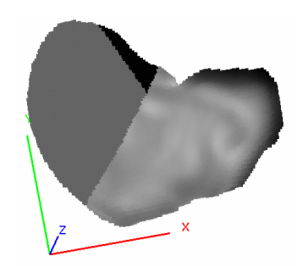

(a)

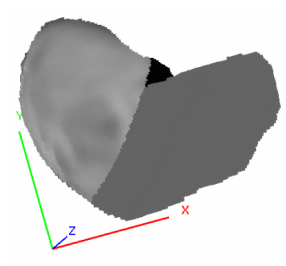

(b)

Fig. 16. (a) Part of segment I contained in the right liver (black). (b) Part of segment I contained in the left liver (black).

\section{$5 \quad$ Experimental Results}

In this section, results obtained by applying the proposed method on 7 clinical cases are presented and analysed.

\subsection{Data set and computation time}

The 3D binary images of the proposed database have been acquired from CTscans and segmented using the the method of Soler et al. [9]. These images present a size varying from $512^{2} \times 100$ to $512^{2} \times 140$ voxels. Voxel spatial dimensions are usually about $0.5 \mathrm{~mm}$, whereas inter-slices separation is about $2 \mathrm{~mm}$. In order to increase the method accuracy and reduce the computation time, the database has been processed to obtain isotropic data composed of $2 \mathrm{~mm}$ edge voxels. On average, liver surfaces are then composed of 21000 voxels.

The computer used for validations was composed of a AMD Athlon(TM) $\mathrm{XP} 1700+, 1.4 \mathrm{GHz}$ processor and $512 \mathrm{MB}$ of memory. The computation of curvature images from the binary liver image requires less than 1 minute. Moreover, this time can be reduced by using the "sliding window" technique [11], which consists in handling the in-going and out-going voxels of the structuring element during the computation. The computation time of the liver segment delineation process requires less than 6 minutes.

\subsection{Results and discussion}

Measuring the relevance of results of the proposed method is not a trivial task, since it is impossible to compare the resulting segments with the real anatomical ones. Indeed, the methods of plane placement only provide an 
approximation of the real liver segmental structure [12]. Furthermore the real segments for each clinical case of the database are not available. The only possible measure consists then in evaluating the position of the automatically computed planes in comparison with human manual positioning, but even in this field, different experts do not necessarily agree. Hence, to estimate the accuracy of automatic plane placement, the following protocol is used. Two experts have interactively placed 5 planes according to the portal and hepatic venous distribution patterns using the $3 D$ Virtual Surgery Planning software [13]. The position of the automatic computed planes and the position of the planes chosen by the experts have been compared. A comparison of the difference between the results of both experts also provides an approximation of the relevance of their positioning.

The error metrics are composed of distances and volume difference ratios which have been computed from the 7 clinical cases. The distance between planes is computed, using pseudo-metrics [14], as follows. For each point within a plane, the distance to the nearest point in the other plane is computed. The position of a plane is then quantified by a set of minimal and maximal distances. The maximum of these distances is the Hausdorff distance. To complete these metrics, the volume of error between each couple of planes which corresponds to the number of voxels attributed to different segments is also computed. For plane ABC, which delineates the left and the right hemiliver, the error is measured by dividing the number of miss-classified voxels by the total liver volume (see Fig. 17(a)). The same parameter is used for plane ABD, which delineates the left from the right liver lobe. For plane A'B'E splitting segment II from segment III, the error is only measured on the common area composed of segment II and segment III. In this case, the number of voxels of the common area attributed to different segments is divided by the volume of this area (see Fig.17(b)). This restriction is useful because it avoids the accumulation of errors. Otherwise the error involving planes $\mathrm{ABC}$ and $\mathrm{ABE}$ would be measured. The same restricted metrics are used for planes ABF and HH'F in the right hemiliver.

The mean error values between each expert and the method, and between both experts are summarized in Table 1. One can observe that the values for planes $\mathrm{ABC}$ and $\mathrm{ABD}$ are relatively correct in comparison to the errors between both experts (Exp1-Exp2 column). This can be explained by the fact that the construction of these planes is based on 3 external anatomical landmarks which are efficiently detected by the method. In fact, points A and $\mathrm{B}$ have been chosen on the cave trunk which is a very reliable landmark. Furthermore, the point $\mathrm{C}$ is on the bed of the gall bladder and the point $\mathrm{D}$ is on the trace of the suspensory ligament, which are the only external landmarks strongly correlated with reliable internal vascular landmarks. Values obtained for plane ABF are also good. One can consider that this plane is acceptable in comparison to the values between both experts. The results are a little less 


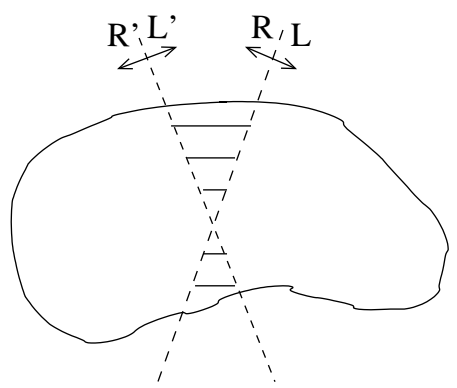

(a)

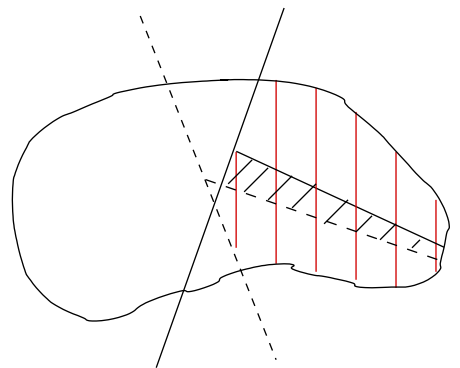

(b)

Fig. 17. Principle involved in the measurement of volume errors between two planes. (a) The dashed area is the volume of miss-classified voxels. (b) For plane ABE, only the volume errors between planes in the common subsegmental area composed of segment II and segment III (vertical dashes) is considered. The error is represented by the area with slanted dashes.

satisfactory since this plane is built from 2 reliable points (A and B) and point $\mathrm{F}$, which is only a geometrical landmark.

It has to be noticed that these 3 planes are the 3 vertical separation planes according to the guidelines currently used in radiological practice. The error induced by these 3 planes on segment evaluation by comparison to the real segmental structure has been studied by Fasel et al. in [12]. By computing the error ratio between flat radiological planes and real boundaries, they obtained a mean error value for 5 clinical cases of $34 \%$ with a minimum of $5.6 \%$ and a maximum of $86 \%$. By using the same evaluation approach concerning the 3 planes, the obtained average difference between the proposed method and the 2 experts is respectively $17 \%$ and $28 \%$. However, it is impossible to affirm that these results are more satisfactory than those proposed in [12], since the expert plane positions can not be considered as the anatomical reality. This assumption is strengthened by the $23 \%$ difference between both expert results.

Values obtained for planes A'B'E and HHF present errors. Indeed, for these planes, the difference between the method and each expert is higher than previously, while the evaluations of both experts are similar. This can be explained by the fact that experts use vessels of portal network for the definition of these planes, whereas the automatic method only uses geometrical criteria. It seems necessary to improve the method in further works in order to automatically detect the same internal landmarks as experts. For A'B'E the median point located between the separation of PR and LL veins and the Rex's recessus could be used. Then, it could be possible to build a plane passing through that point and being parallel to the coronal plane. The HH'F transversal plane placement could be improved by passing through the separation point between the PR vein and the $L R$ vein and parallel to the axial plane. 


\begin{tabular}{|c|c|c|c|c|}
\hline \multicolumn{5}{|c|}{ Errors } \\
\hline Plane & & Exp1-Auto & Exp2-Auto & Exp1-Exp2 \\
\hline \multirow[t]{3}{*}{$\mathrm{ABC}$} & $\min$ & $0.37 \pm 0.64$ & $0.38 \pm 0.99$ & $0.79 \pm 2.09$ \\
\hline & $\max$ & $24.60 \pm 22.62$ & $32.22 \pm 20.04$ & $24.66 \pm 14.82$ \\
\hline & $\%$ vol & $3 \pm 2$ & $7 \pm 4$ & $5 \pm 3$ \\
\hline \multirow[t]{3}{*}{$\mathrm{ABD}$} & $\min$ & $10.25 \pm 17.51$ & $5.04 \pm 7.52$ & $7.89 \pm 15.81$ \\
\hline & $\max$ & $30.03 \pm 27.11$ & $42.02 \pm 19.95$ & $52.11 \pm 35.15$ \\
\hline & $\%$ vol & $4 \pm 6$ & $8 \pm 5$ & $8 \pm 5$ \\
\hline \multirow[t]{3}{*}{$\mathrm{ABF}$} & $\min$ & 0.00 & 0.00 & $0.7 \pm 1.85$ \\
\hline & $\max$ & $53.74 \pm 14.91$ & $55.5 \pm 13.29$ & $50.69 \pm 27.11$ \\
\hline & $\% v^{\prime} l^{\dagger}$ & $17 \pm 2$ & $23 \pm 8$ & $17 \pm 8$ \\
\hline \multirow[t]{3}{*}{$A^{\prime} B^{\prime} E$} & $\min$ & $7.45 \pm 7.26$ & $9.68 \pm 16.89$ & $0.27 \pm 0.70$ \\
\hline & $\max$ & $41.67 \pm 34.47$ & $44.17 \pm 16.26$ & $46.57 \pm 24.23$ \\
\hline & $\% v^{2} l^{*}$ & $18 \pm 8$ & $24 \pm 9$ & $13 \pm 1.5$ \\
\hline \multirow[t]{3}{*}{ HH'F } & $\min$ & $4.33 \pm 4.16$ & $3.14 \pm 5.84$ & 0.00 \\
\hline & $\max$ & $53.27 \pm 7.59$ & $57.99 \pm 7.83$ & $47.14 \pm 18.67$ \\
\hline & $\% v_{o l}^{\dagger}$ & $18 \pm 8$ & $18 \pm 4$ & $11 \pm 4$ \\
\hline
\end{tabular}

Table 1

For each plane and each couple of positioning proposal, the error is characterized by the minimum and maximum distances (in $\mathrm{mm}$ ) and by the ratio of miss-classified voxels $\left(\% v o l=\right.$ ratio over the total liver volume; $\% v_{o l} l^{\dagger}=$ ratio over the common liver right volume; $\% \mathrm{vol}^{*}=$ ratio over the common volume of segments II and III). The errors are presented by the mean value over 7 clinical cases \pm the standard deviation.

\section{Conclusion and perspectives}

In this paper, we have presented a fully automatic method enabling the approximation of anatomical segments of the liver, according to Couinaud's nomenclature, within a short computation time (about 6 minutes). Based on the patient's anatomy, the automated method simulates the strategy of the surgeon. Result evaluations demonstrate its efficiency for the delineation of four liver regions, which results in automatically placing 3 vertical planes according to the radiological guidelines. However, it seems to be less accurate for the placement of the 2 remaining planes. These errors can be explained by the poor correlation between the used geometrical landmarks and the real anatomical ones. Indeed, even though the method by section plane is used 


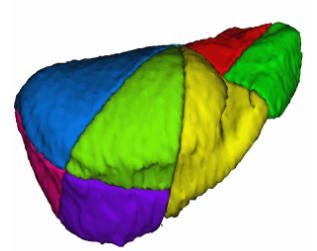

(a)

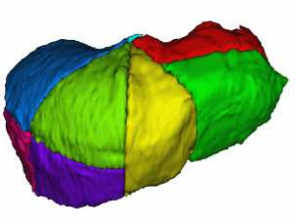

(e)

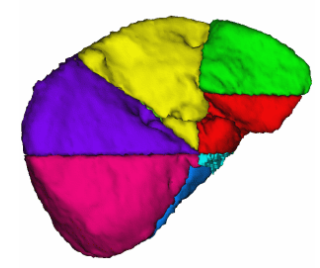

(b)

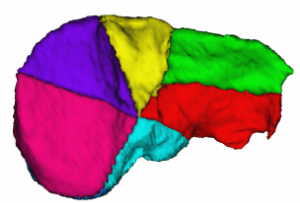

(f)

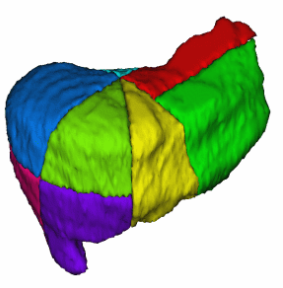

(c)

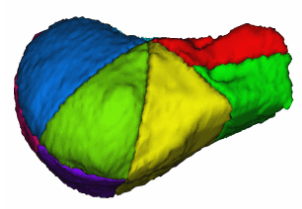

(g)

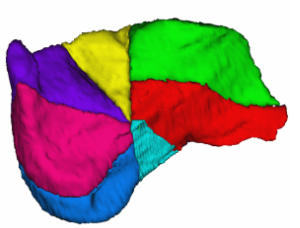

(d)

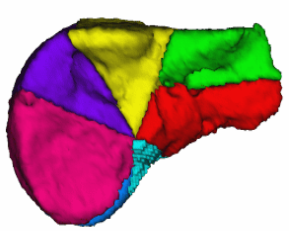

(h)

Fig. 18. Automatically detected liver segments. (a),(c),(e),(g): Top view. (b),(d),(f),(h): Bottom view.

in clinical routine, it remains approximate, as demonstrated in [12]. To improve the segment delineation, it could be useful to complete this computation method based on external landmarks with a method using internal landmarks, i.e. to label the portal vein branches. A first approach could consist in replacing the anatomical atlas in the method of Soler et al. [8] by the resulting segmental model of the proposed method. In particular, if the influence region merging step is guided by the plane localizations the improved method could provide the real segments. We point out that methods by separation planes approximate the anatomical reality, whereas methods based on the study of the vascular network tree are limited by the quality of its reconstruction. Our future research work will then aim at finding a mixed method correlating both approaches of segment computation to use their advantages and to reduce their weaknesses.

Moreover, the promising results obtained on the 3 vertical planes, which are generally defined by hepatic veins, could be used to improve vascular networks segmentation procedures. Indeed, since most scan images are obtained in portal time, hepatic veins are generally difficult to detect. Anatomical knowledge such as the one obtained in the proposed method could then be used for guidance of vessel segmentation. 


\section{References}

[1] C. Couinaud, Le foie, études anatomiques et chirurgicales, Masson, 1957.

[2] N. Goldsmith, R. Woodburne, The surgical anatomy pertaining to liver resection, Surg Gynecol Obstet 105 (3) (1957) 310-318.

[3] H.-P. Meinzer, M. Thorn, C. C. S., Computerized planning of liver surgery An overview, Computers \& Graphics 26 (4) (2002) 569-576.

[4] G. Glombitza, W. Lamadé, A. M. Demiris, M.-R. Gpfert, A. Mayer, M. L. Bahner, H.-P. Meinzer, G. Richter, T. Lehnert, C. Herfarth, Virtual planning of liver resections: image processing, visualization and volumetric evaluation, International Journal of Medical Informatics 53 (2-3) (1999) 225-237.

[5] A. Osorio, P.-J. Valette, A. Mihalcea, J. Atif, X. Ripoche, A new PC based software to perform semi-automatic hepatic segmentation using CT or MR images, in: InfoRAD RSNA 2002, 88th Annual Meeting of the Radiological Society of North America, Chicago, USA, November 13-December 6, 2002, Proceedings, 2002.

[6] D. Selle, B. Preim, A. Schenk, H.-O. Peitgen, Analysis of vasculature for liver surgery planning, IEEE Transactions on Medical Imaging 21 (11) (2002) 13441357.

[7] D. Selle, W. Spindler, B. Preim, H.-O. Peitgen, Mathematical methods in medical imaging: analysis of vascular structures for liver surgery planning, in: B. Enquist, W. Schmid (Eds.), Mathematics Unlimited - 2001 and Beyond, Springer, 2000, pp. 1039-1059.

[8] L. Soler, Une nouvelle méthode de segmentation des structures anatomiques et pathologiques : application aux angioscanners $3 \mathrm{~d} d u$ foie pour la planification chirurgicale, Ph.D. thesis, université de Paris XI, Orsay (1998).

[9] L. Soler, H. Delingette, G. Malandain, J. Montagnat, N. Ayache, C. Koehl, O. Dourthe, B. Malassagne, M. Smith, D. Mutter, J. Marescaux, Fully automatic anatomical, pathological, and functional segmentation from CT scans for hepatic surgery, Computer Aided Surgery 6 (3) (2001) 131-142.

[10] J. Bullard, E. Garboczi, W. Carter, E. Fuller, Numerical methods for computing interfacial mean curvature, Computational Materials Science 4 (2) (1995) 103116.

[11] M. Van Droogenbroeck, H. Talbot, Fast computation of morphological operations with arbitrary structuring elements, Pattern Recognition Letters 17 (14) (1996) 1451-1460.

[12] J. Fasel, D. Selle, C. Evertsz, F. Terrier, H.-O. Peitgen, P. Gailloud, Segmental anatomy of the liver: poor correlation with CT, Radiology 206 (1) (1998) 151156. 
[13] C. Koehl, L. Soler, J. Marescaux, A PACS based interface for 3D anatomical structures visualization and surgical planing, in: S. Mun (Ed.), Medical Imaging 2002: Visualization, Image-Guided Procedures, and Display, San Diego, CA USA, February 23-March 1, 2002, Vol. 4681, SPIE, 2002, pp. 17-24.

[14] V. Chalana, Y. Kim, A methodology for evaluation of boundary detection algorithms on medical images, IEEE Transactions on Medical Imaging 16 (5) (1997) 642-652. 